significant reductions in GLUT3 levels which correlated with greater amyloid- $\beta(A \beta)$ and neurofibrillary tangle pathological burden in participants with $\mathrm{AD}$ pathology at post-mortem but without evidence of cognitive dysfunction in their lifetime. Some studies showed increased GLUT1, with others showing reduced GLUT1, levels in AD brain. A newly recognised GLUT12 appears to be increased in AD. Animal studies showed similar results with GLUT1 and GLUT3 knockout animal models exhibiting AD pathology, while overexpression of GLUT1 or treatment with metformin decreased $A \beta$ toxicity in a Drosophila model of AD. GLUT2 levels were increased in both human $\mathrm{AD}$ brain and in an animal model of $\mathrm{AD}$. Imaging studies using fluorodeoxyglucose [18F]FDG with positron emission tomography (FDG-PET) in AD subjects show reductions in glucose transport and glucose metabolism in areas most affected in $\mathrm{AD}$. A small randomised control trial showed anti-diabetic medications improved the glucose transport in AD subjects.

Conclusion. GLUTs play a significant role in AD pathology with evidence suggesting that GLUT3 reductions may precede the onset of clinical symptoms, while GLUT2 and GLUT12 may have a compensatory role. Repurposing anti-diabetic drugs shows promising results in both animal and human studies of $\mathrm{AD}$.

Battered woman syndrome and PTSD in women who kill their abusing partner: a study in medical jurisprudence

Sharmilaa Lagunathan

Leicester Medical School, University of Leicester

doi: 10.1192/bjo.2021.708

Aims. The aim of the study was to identify any symptoms or features of Battered Woman Syndrome (BWS) or Post-traumatic Stress Disorder (PTSD) that may be associated with, or explain, abused women killing their abuser; and the extent to which such identified symptoms or features have been deemed, or are potentially relevant, to past and now reformed partial defences to murder in English law. Hence two sub-studies were completed. Method. The first sub-study identified mental symptoms of BWS or PTSD apparent in battered women who kill their abuser; achieved by identifying relevant research papers, through applying a 'rapid review' approach to three databases: PubMed, PsychInfo and PsychArticles. The second sub-study identified by legal research reported Court of Appeal (CA) judgments on women appealing their conviction of the murder of their abusive partner. It then analysed the legal approach taken towards evidence of the effects of abuse upon these women before and after relevant statutory law reform (although no CA cases were identified post-reform).

Result. The first sub-study identified and reviewed six symptoms or features, within three quantitative and three qualitative studies, that appeared to be associated with, or described by, abused women killing their abuser. These included helplessness, symptoms associated with PTSD, plus fear, isolation, experience of escalation of violence and cycle of violence. From the CA cases the perpetrators of killings that occurred prior to 04.10.2010 (the date of law reform) were usually successful in having their conviction overturned based upon diminished responsibility; but not provocation, because of the requirement of 'sudden loss of self control'. 'Loss of control', which replaced provocation, appears highly likely to be capable of reducing murder to manslaughter based upon symptoms of BWS, or PTSD. However, the amended defence of diminished responsibility is likely to exclude evidence of BWS, but allow evidence of PTSD, because of its requirement of the defendant suffering from 'a recognised medical condition'.

Conclusion. This study demonstrated particular symptoms or features of BWS or PTSD associated with abused women killing their abusers plus their very different relevance to two partial defences to murder, pre and post law reform.

\section{Alcohol use disorder and its association with personal well-being and life satisfaction}

Chau Sian Lim, Zhen Wei Lew, Yoke Chiang Ng, Lai Huat Peh and Hatta Santoso Ong

Changi General Hospital

${ }^{*}$ Corresponding author.

doi: 10.1192/bjo.2021.709

Aims. This study aims to find out how alcohol use disorder (AUD) correlates to personal well-being and life satisfaction.

Background. AUD is prevalent and leads to significant physical, physiological, and social-occupational impairment. Mental wellbeing involves the overall positive psychological state of a person being well adjusted, socially engaged, and emotionally healthy. Despite the paradigm shift from purely treating mental illness to promoting positive mental health, there is limited literature describing the relationship between alcohol use disorder and mental well-being.

Method. This cross-sectional study was conducted in a general hospital in Singapore. Patients admitted across a span of two years were screened for possible alcohol use disorder. Patients were included if they were male, aged 21 years and above, and had the mental capacity to give consent. They were excluded if they had illicit drug use, acute mental illness, inability or refusal to give consent, or if they were already receiving intervention for addiction issues. Participants were administered the Alcohol Use Disorders Identification Test (AUDIT). Those who scored 8 or above were classified as being at risk for AUD, while those who scored 7 or less were classified as at low risk. They were also administered the Personal Wellbeing Index (PWI) and the "Satisfaction with Life as a Whole" question. The PWI measures individuals' subjective well-being across seven domains. The "Satisfaction with Life as a Whole" question measures, on an eleven-point Likert scale, how satisfied the respondent feels with life in general. Demographic data were also collected and STATA v. 12.1 was used for statistical analysis.

Result. Among a total of 134 participants, 25 of them scored $\geq 8$ on the AUDIT and 109 scored 7 or less. On the PWI, the group at risk scored significantly lower at 71.3 (95\% CI: 66.0-76.7) compared to the group not at risk at 77.9 (95\% CI: 75.8-79.9), p < 0.01 . The results were similar on the "Satisfaction with Life as a Whole" item. The group at risk had a mean of 6.72 (95\% CI: $6.03-7.41)$ while the group not at risk had a mean of $7.67(95 \%$ CI: 7.41-7.93), both $\mathrm{p}<0.01$. The differences between the high risk and low risk groups remained statistically significant even after adjusting for differences in age, race, education level, and employment status.

Conclusion. This study demonstrated a statistically significant association between AUD and personal well-being as well as satisfaction with life among males. 